\title{
Interactive comment on "Examining controls on peak annual streamflow and floods in the Fraser River Basin of British Columbia" by Charles L. Curry and Francis W. Zwiers
}

\section{Charles L. Curry and Francis W. Zwiers}

cc@uvic.ca

Received and published: 2 December 2017

We thank Dr. Dery for his insightful and helpful review of our manuscript. In response to the general comments made in his review, we offer the following:

1) Paper structure: In response to this criticism, and acknowledging similar comments from Reviewer 2, we have reorganized the content in the revised manuscript. Specifically, the descriptions of methods in the Results were moved to Section 2: Data and Methods.

2) We thank the referee for mentioning this additional data set for SWE. We agree that,

Printer-friendly version

Discussion paper 
were the focus of the paper on the predictability of APFs in an operational sense, i.e. flood forecasting, then incorporating these additional data would be a priority. However, as now more clearly stated at several points in the paper, the intent of our work is rather different. Namely, we aim to discover and quantify relationships between the interannual variability of APF, large-scale climate indices, and various basin-averaged variables within the FRB. We feel that the data that we have used are adequate for this purpose; the MSS SWE data already demonstrate a strong connection between the annual max(SWE) and APF, and thus uncertainty in annual max(SWE) does not seem to be a significant factor.

3) The selection of subbasins was made with three objectives in mind. The first was that all subbasins together capture a large fraction of the resultant flow at Fraser-Hope. As mentioned in the Abstract and in the first sentence of Sec. 3.6, the chosen subbasins collect nearly $70 \%$ of the observed mean annual streamflow at Fraser Hope (note that a reference to Kang et al. 2016, which provides the $70 \%$ estimate, has been added to the sentence). The second objective was to select basins that are sufficiently varied that they represent a range of elevations and, if possible, streamflow regimes. While the three larger subbasins are located in the eastern part of the FRB and represent nival environments, the Chilko basin lies on the westward edge of the FRB and integrates a significant amount of rainfall falling on the east-facing side of the Coast Mountains, making it a hybrid (nival-pluvial) environment. It was important to include this subbasin in order to probe the sensitivity of outlet streamflow to rainfall (although no such influence was detected on the APF in the Chilko). The third consideration was to avoid regulated basins, of which the Nechako-Nautley is one. In summary, although some medium-sized subbasins with sufficiently long data records were omitted from the study, we think it is unlikely that any important qualitative features of the climateAPF relationship were missed as a consequence.

4) We thank the referee for raising this thought-provoking point. We did not attempt to quantify the contribution of each subbasin to the overall APF at Fraser-Hope, nor did we

Printer-friendly version

Discussion paper
Interactive

comment 
calculate the lag between the respective peak flow days. We agree that information on the timing and magnitude of upstream flows would likely serve as effective predictors of APF at Fraser-Hope. However, as mentioned in point 2) above, the focus of our study is primarily on the climate-APF relationship on the interannual time scale, rather than short term prediction or monitoring of the upstream flow. We take this perspective not only to clarify the influence of historical climate variables on APF, but also to provide guidance for further study of the influence of changing climate on APF.

Regarding the specific comments raised by Dr. Dery:

1) We have added a sentence to the first paragraph of the Introduction pointing out the significance of the FRB for the salmon fishery in BC.

2) We have endeavoured to use abbreviations consistently after their first introduction in the manuscript.

3) We have removed this information from the Introduction, since it is already provided at the beginning of Section 2.

4) We agree with the referee and have removed the Dery et al. (2012) reference from this sentence, which refers mainly to the more recent paper. The former reference still appears in the next sentence.

5) Change made.

6) Noted, we will seek the editor's instruction on date formats for HESS.

7) We have corrected this and hopefully all other instances of missed unit superscripts in the revised ms.

8) Noted and changed, thank you.

9) Year added to this reference.

10) We have defined these abbreviations in the text. 
11) The sentence concerning VIC elevation bands has been redrafted as follows: "The VIC implementation used in this study incorporates five elevation bands corresponding to $200 \mathrm{~m}$ vertical resolution, with the number of elevation bands in any one $1 / 16^{\circ}$ grid cell depending on the topography within that cell."

12) We have added the suggested references to our initial discussion of teleconnections on p. 3.

13) Done.

14) As stated in the caption to Table 3 , the trends in variables were calculated using Sen's median slope estimator, which is more robust to outliers than the standard leastsquares method. This correction has been inserted at the beginning of Sec. 3.1.

15) We thank the referee for pointing out that most sites in the Upper Fraser studied in their previous paper have annual maximum SWE dates later than April 1. For our study, we only had access to the April 1 values from the MSS data, having obtained these data from a secondary source (Dr. R. Najafi). Since this was not made clear in the submitted MS, we have altered the data description in Sec. 2.1 to reflect this. More substantively, although using April 1 values might underestimate the true annual max(SWE), we do not believe this would bias the regression of $\max (\mathrm{SWE})$ versus APF, since years with larger/smaller than normal April 1 SWE should also feature larger/smaller than normal true $\max (\mathrm{SWE})$. Since it is the sign and magnitude of the annual max(SWE) anomaly, rather than its absolute value, that enters the regression analysis, we doubt that this would significantly alter the strong relationship found between observed SWE and APF discussed in Sec. 3.3.1.

16) Yes, change made.

17) Units have been inserted in Eqs. (1)-(3) (addressing point 19) also).

18) p-values inserted, thank you.

Printer-friendly version

20)-25), 27) These changes were made.

Discussion paper

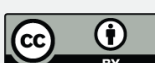


26) The DOI has been added to this entry.

28) Our justification for the choice of subbasins was provided in the response to point 15) above. The period of record for each subbasin as listed in Table 1 reflects data availability from the WSC at the time of the analysis. However, note that observed discharge was only analyzed for the Fraser-Hope station, and not for the subbasin outlets.

29) The surface routing scheme in the VIC model does not account for any regulation of flows occurring in the real FRB. Consequently, the calibration procedure uses naturalized flow estimates in subbasins such as the Nechako where such diversions occur. For this reason, we can be confident that the difference between the mean hydrographs displayed in Fig. 5b are not attributable to this effect. Indeed, the difference is so small compared to the large interannual variability in both observed and VIC-simulated hydrographs that we are reluctant to conclude that the hydrographs are, in fact, significantly different.

Interactive comment on Hydrol. Earth Syst. Sci. Discuss., https://doi.org/10.5194/hess-2017531, 2017. 